\title{
REFLUJO VESICOURETERAL GRADOS III-IV: FACTORES IMPLICADOS EN LA EFECTIVIDAD DEL TRATAMIENTO ENDOSCÓPICO EN PACIENTES PEDIÁTRICOS.
}

\author{
Roberto Méndez, Ivan Somoza, Manuel G. Tellado, Jorge Liras, Alberto Sánchez-Abuín, \\ Ernesto Pais y Diego Vela.
}

Servicio de Cirugía Pediátrica. Complexo Hospitalario Universitario "Juan Canalejo". La Coruña. España.

\begin{abstract}
Resumen.- OBJETIVO: El Reflujo Vesicoureteral (RVU) es una de las anomalías congénitas urológicas más frecuentes y se detecta hasta en el 30-50\% de los pacientes pediátricos diagnosticados de Infección del Tracto Urinario (ITU). Su tratamiento es controvertido. Existen múltiples estudios clínicos que comparan los resultados de la terapéutica médica antibiótica frente a la reimplantación quirúrgica, pero desde la introducción de una tercera alternativa en el año 1984 (tratamiento endoscópicol, las recomendaciones y protocolos de tratamiento del RVU han cambiado dramáticamente. El objetivo de nuestro estudio es el de describir y analizar los factores que influyen en la curación del reflujo mediante tratamiento endoscópico en sistemas con RVU grados III y IV según la Clasificación Internacional.
\end{abstract}

MÉTODOS: Estudio de cohortes retrospectivo y prospectivo que analiza los 90 pacientes con RVU grados III y IV tratados endoscópicamente entre marzo de 1998 y diciembre de 2004. Sobre un total de 550 pacientes (735 unidades renales refluyentes) diagnosticados de

\footnotetext{
Roberto Méndez Gallart Servicio de Cirugía Pediátrica Complexo Hospitalario de Santiago Travesía da Choupana, s/n 15706. Santiago de Compostela. la Coruña. (España) roberto.mendez.gallarł@sergas.es Trabajo recibido: 12 de diciembre 2005
}

RVU en el Servicio de Cirugía Pediátrica de A Coruña en el período de estudio, se seleccionaron sólo 184 tratados endoscópicamente. El grupo final de estudio de 90 pacientes (1 30 unidades) se constituyó tras excluir los casos de reflujo secundario /vejiga neurógena, ureterocele) y aquellos con seguimiento incompleto. Todos los pacientes fueron sometidos a cistografía miccional convencional o bien a Sonocistografía con galactosa para realizar el diagnóstico, gammagrafía con DMSA para evaluar la nefropatía, ecografía renal y vesical y exploración clínica del patrón miccional. Se registraron datos sobre sexo, edad, ITU, bilateralidad, daño renal, disfunción miccional, tasa de curación, complicaciones y recurrencias. Definimos un nuevo factor: grado de dilatación ureteral independiente de la Clasificación Internacional. Este factor fue valorado por un único urólogo pediátrico sin conocer el grado de la Clasificación Internacional. Los datos fueron analizados empleando el test estadístico del chi-cuadrado y el test exacto de Fisher además de una regresión logística múltiple. Se estableció significación estadística con valores de $p<0.05$. Empleamos el paquete estadístico SPSS 11.0 para el estudio de los datos.

RESULTADOS: La relación niños/niñas fue de 2.91. El RVU fue bilateral en $44.4 \%$. UTI se diagnostico como forma presentación en 64 casos y el diagnóstico fue antenatal en 21 (con predominio de varones 5.2:1). Encontramos una relación estrecha entre la incidencia de daño renal en el momento del diagnóstico y existencia de disfunción miccional $(p<0.01)$. La tasa de curación con la primera inyección fue del 64\%. Mientras que en análisis univariable mostró una fuerte relación entre la curación con la $1^{\underline{a}}$ inyección y la bilateralidad, disfunción miccional, nefropatía inicial y grado de dilatación ureteral, la regresión logístico multivariante el único factor realmente significativo a la hora de predecir la respuesta al tratamiento fue la existencia de una dilatación ureteral severa frente a una dilatación 
leve ( $p<0.001$; odds ratio 0.045, IC 95\% 0.01-0.16). La progresión del daño renal fue detectada especialmente en aquellos pacientes con disfunción miccional $(p=0.053)$, bilateralidad ( $p=0.034)$ y dilatación ureteral severa $(p<0.001)$.

CONCLUSIONES: Se demuestra la relación directa entre la tasa de curaciones con el tratamiento endoscópico del los reflujos grados III y IV y el grado de dilatación ureteral. Otros factores relevantes implicados en la resolución endoscópica del RVU son la existencia de disfunción miccional, bilateralidad y existencia de daño renal medido por DMSA en el momento del diagnóstico inicial. El sexo, edad y número de infecciones durante el seguimiento no tienen influencia en el pronóstico de curación del reflujo de grados III y IV tratado mediante el uso de sustancias biocompatibles a nivel subureteral.

Palabras clave: Reflujo Vesicoureteral. Tratamiento endoscópico. Nefropatía. Pediatría.

Summary.- OBJECTIVES: Vesicoureteral reflux (VUR) is detected in $30-50 \%$ of pediatric patients diagnosed of urinary tract infection (UTI). Multiple clinical trials have compared the results of conservative management against surgical therapy. The introduction of a third alternative in 1984 (endoscopic approach) has dramatically modified the management of VUR. The objective of our work is to analyze the factors influencing the favourable outcome of the endoscopic treatment of VUR

METHODS: Retrospective and prospective cohort study analyzing 90 patients with grades III and IV VUR treated endoscopically over the last eight years. 184 patients undergoing endoscopic management were selected from a group of 550 patients (735 VUR renal units) with the diagnosis of VUR over the study period, . All patients underwent VCUG or galactose-based sonocystography to obtain the diagnosis. DMSA scintigram was used to evaluate renal damage. Data about gender, age, UTI, bilateralism, renal damage, voiding dysfunction, outcomes, complications, and recurrences were registered. A new factor was defined: ureteral dilation independent from the international classification. This factor was evaluated by a single pediatric urologist blinded for the international classification results. Data analysis was performed using the chi square test and Fischer exact test; a multiple logistic regression analysis was also performed. Statistical significance was established at $p$ $<0.05$. SPSS 11.0 software was employed for data analysis.

RESULTS: Boys/girls ratio was 2.91. Bilateral VUR appeared in $44.4 \%$ of the cases. UTI was the presenting diagnosis in 64 cases; the diagnosis was prenatal in 21 cases (with a male predominance 5.2: 1). We found a strong correlation between renal scarring at the time of diagnosis and voiding dysfunction $(p<0.01)$. Cure rate after first injection was $64 \%$. Although univariate analysis showed a strong correlation between cure and first injection, bilaterality, voiding dysfunction, initial nephropathy, and ureteral dilation grade, on multivariate logistic regression the only significant factor to predict treatment response was the existence of severe ureteral dilation in comparison with mild dilation $(\mathrm{p}<0.001$; odds ratio $0.045,95 \%$ confidence interval 0.01-0.16). Renal damage progression was specially detected in those patients with voiding dysfunction ( $p=0.053)$, bilaterality $(p=$ $0.034)$ and severe ureteral dilation $(p<0.001)$.

CONCLUSIONS: A direct relation between cure rate after endoscopic treatment of grade III and IV reflux and severity of ureteral dilation was demonstrated. Other relevant factors implied in the endoscopic resolution of VUR include the existence of voiding dysfunction, bilateral reflux and renal damage determined by DMSA at the time of initial diagnosis. Gender, age, and number of infections during follow up have no influence in the probability of cure of grade III and IV VU reflux treated with subureteral injection of biocompatible substances.

Keywords: Vesicoureteral reflux. Endoscopic treatment. Nephropathy. Children.

\section{INTRODUCCIÓN}

El Reflujo Vesicoureteral afecta al 30-50\% de los pacientes pediátricos con infección del tracto urinario. La asociación entre reflujo, infección urinaria y daño renal está bien establecida. La nefropatía por reflujo es la causa de insuficiencia renal terminal en el $3-25 \%$ de los niños y del $10-15 \%$ de los adultos $(1,2)$.

Si bien el diagnóstico, la fisiopatología y la clasificación del RVU han sido estandarizados, el tratamiento de esta patología es controvertido. Tras el consenso establecido en 1981 para la Clasificación Internacional de los diversos grados de reflujo, se planteó el tratamiento más idóneo para cada grado, estableciendo una serie de normas básicas mundialmente aceptadas. Entre esta serie de recomendaciones se citaba el tratamiento antibiótico profiláctico electivo para aquellos reflujos de bajo grado (I y II) mientras que se aconsejaba el tratamiento quirúrgico para los reflujos de grado $V(3)$. Aunque no se ha demostrado en ninguna publicación que uno $u$ otro tratamiento sean más efectivos a la hora de evitar la aparición de cicatrices renales, sí que se ha comprobado como el número de infecciones urinarias dismi- 
nuye en aquellos pacientes que han sido intervenidos quirúrgicamente (4).

La controversia surgía en torno a los reflujos grados III y IV. Múltiples estudios se han centrado únicamente en analizar la conveniencia del tratamiento médico frente al tratamiento quirúrgico en estos casos seleccionados (5-7). Pero debido a la ausencia de diferencias entre ambos grupos no lograron concluir una recomendación standard en el manejo de estos pacientes. Sabemos que con profilaxis antibiótica, los reflujos de grados III y IV tiene una tasa espontánea de curaciones del $27 \%$ el primer año y del $52 \%$ tras 10 años de tratamiento, mientras que de forma global el tratamiento endoscópico consigue tasas del $77 \%$ (8). Pero la cuestión clave de la terapéutica del reflujo sigue sin resolverse: ¿Cuál es el objetivo del tratamiento del RVU? No existe un consenso entre los especialistas en reflujo acerca de si el objetivo del tratamiento se centra en eliminar totalmente el reflujo o únicamente evitar el daño renal: si lo que pretendemos es curar el reflujo, no existe lugar a dudas respecto a que el mejor método de tratamiento en reflujos III y IV es el endoscópico (efectivo y poco agresivo), pero si el objetivo es evitar el daño renal, ninguna de las tres opciones terapéuticas ha demostrado ser superior a las otras y por lo tanto cualquier actitud es válida.

El objetivo fundamental de nuestro trabajo es el de analizar nuestra serie de pacientes pediátricos con Reflujo Vesicoureteral de alto grado (grados III y IV) tratados endoscópicamente y buscar los parámetros que nos orienten acerca de la probabilidad de obtener el éxito terapéutico.

\section{MATERIAL Y MÉTODOS}

Para la realización de nuestro estudio clínico observacional de cohortes retrospectivo y prospectivo recurrimos a una muestra de pacientes pediátricos diagnosticados de RVU Primario sometidos a tratamiento endoscópico desde marzo 1998 hasta diciembre 2004. Los Criterios de inclusión en la muestra fueron:

\section{Edad entre 1 día y 14 años;}

2.Reflujo vesicoureteral primario unilateral o bilateral;

3.Reflujos de grado III o IV según la Clasificación Internacional;

4.Seguimiento completo por parte del Servicio de Cirugía Pediátrica;

5.Tratamiento endoscópico en algún momento del seguimiento;

6. Estudios de imagen cistográficos adecuados;

7. Documentación clínica completa. Excluímos aque- llos pacientes que presentaban:

1.RVU secundario (ureterocele, válvulas de uretra posterior);

2.Insuficiencia renal en el momento del diagnóstico;

3.Falta de seguimiento a largo plazo;

4.Diagnóstico de Mielomeningocele;

5.Duplicidad ureteral completa.

De un total de 550 casos de RVU 1735 unidades renales refluyentes) diagnosticados en nuestro Hospital en este período, seleccionamos 184 pacientes sometidos a tratamiento endoscópico en algún momento de su seguimiento. Del total de 184 pacientes se seleccionaron en base a los criterios de inclusión y exclusión, 90 casos (130 unidades renales refluyentes) con un seguimiento medio de 19.2 meses ( 6 meses -6 años).

Todos los pacientes de la serie fueron sometidos al menos a una ecografía, cistografía miccional y gammagrafía con DMSA. En los últimos 2 años hemos generalizado el empleo de la sonocistouretrografía miccional con galactosa para el diagnóstico y seguimiento de los pacientes con RVU. Una vez confirmado el diagnóstico de RVU mediante cistografía convencional o sonocistografía con galactosa, se estratificaron los pacientes en 5 grupos según la Clasificación Internacional del reflujo. Los pacientes con RVU de grado I fueron considerados candidatos a tratamiento médico. Los reflujos de grado II inicialmente se trataron de forma conservadora, si bien fueron candidatos a corrección endoscópica siempre que, tras un mínimo de 1 año de tratamiento, no se comprobase su mejoría en una cistografía. Los reflujos de grado III y IV fueron considerados inicialmente susceptibles de tratamiento médico y/o endoscópico según la bilateralidad y la existencia o no de nefropatía. Aquellos reflujos de grado III unilaterales y sin daño renal se trataron médicamente durante un año. Si había evidencia de lesión renal en el momento del diagnóstico, o bien si el RVU era bilateral, se optó por un tratamiento endoscópico. En los pacientes susceptibles de tratamiento médico se repitió la cistografía y DMSA a los 12 meses del inicio. Si el reflujo permanecía o había disminuído a grado I sin aparición de nuevas cicatrices renales se suspendía el tratamiento y se consideraba curación. De forma excepcional, cuando un paciente presentaba un RVU grado IV bilateral y nefropatía del reflujo severa la reimplantación fue la técnica indicada de forma inicial. En aquellos pacientes con RVU grado $V$ se realizó la reimplantación quirúrgica (Técnica de Cohen).

El procedimiento endoscópico de inyección de polidimetilsiloxano -PDMS- (microburbujas de silicona) que empleamos actualmente en nuestro Servicio se realiza mediante anestesia general y posición 
de litotomía del paciente. El material se introduce mediante una pistola de alta presión. Se introduce la aguja aproximadamente $3 \mathrm{~mm}$ bajo el meato de forma submucosa y se inyectan 0.2-0.6 cc de PDMS (Macroplastique ${ }^{\circledR}$, Uroplasty Inc, Geleen, The Netherlands) hasta comprobar la aparición de un abultamiento en forma de volcán. La aguja se mantiene en la misma posición durante unos 30 segundos para evitar la extrusión inmediata del producto. A las 8 horas del procedimiento se da de alta hospitalaria al paciente sin precisar medicación analgésica.

Analizamos las siguientes variables clínicas: Edad, Sexo, Forma de presentación clínica, Grado de reflujo según la Clasificación Internacional, Grado de dilatación ureteral aislada lla graduación se realizó mediante un estudio de doble ciego con dos especialistas en Urología Pediátrica que valoraron de forma aislada la dilatación del uréter sin conocer la graduación previa del sistema ni el resultado del tratamiento endoscópico), Nefropatía del reflujo según el DMSA, Disfunción miccional, Porcentaje de curación del reflujo, Progresión de la nefropatía, Complicaciones y recurrencias.

Se realizó un estudio descriptivo de todas las variables incluidas en el trabajo. De las Variables cuantitativas se calcularon las medidas habituales de centralización y dispersión (media, mediana, rango y desviación standard) con sus intervalos de confianza del $95 \%$. Para las Variables cualitativas se expresan su valor absoluto, porcentaje e intervalo de confianza con una seguridad del $95 \%$ (Fleiss). Tras determinar la normalidad o no de las variables cuantitativas con el test de Kolmogorov-Smirnov, se utilizó para la comparación de medias la $t$ de Student de datos independientes o el test $U$ de Mann-Whitney si las condiciones del t-test no se verificaban. Para la comparación de variables cuantitativas en más de dos grupos de pacientes se utilizó un análisis de la varianza ANOVA o el test de Kruskall-Wallis. Para la asociación de variables cualitativas se utilizó el chicuadrado $\left(x^{2}\right)$ de Pearson o el test exacto de Fisher y la estimación del odds ratio y su $95 \%$ intervalo de confianza. Si las variables cualitativas presentaban una escala ordinal, aparte del chi-cuadrado se calculó la d de Somer. Para el estudio de la correlación de las variables cuantitativas se calculó el coeficiente de correlación de Spearman.

Identificadas las variables que en el análisis univariante se asociaban o no a la resolución de reflujo, se realizó un análisis de regresión logística múltiple. El nivel de significación estadística $\alpha$ establecido en todos los análisis fue de $p<0.05$. Los test estadísticos han sido realizados con un planteamiento bilateral. El análisis de los datos se llevó a cabo con el programa estadístico SPSS ${ }^{\circledR} 11.0$ (Statistical Package for Social Sciences versión 11.0).

\section{RESULTADOS}

El $74.4 \%$ (67) eran niñas y el $25.6 \%$ niños (23). La razón de feminidad o cociente niñas / niños fue 2.91. Esto supone que en nuestra serie el reflujo de grados III y IV es casi 3 veces más frecuente en niñas que en varones. El rango de edades al diagnóstico abarcó desde la época prenatal (con cistografía realizada en la primera semana de vida) hasta los 13 años. La edad media fue de 2.8 años con una desviación típica de 3.3 años y una mediana de 1 año. La gran mayoría de los pacientes de la serie fueron diagnosticados tras la realización de una cistografía después de una infección del tracto urinario. El $23 \%$ de los pacientes se diagnosticaron tras haber detectado una hidronefrosis antenatal en las ecografías fetales. El $86 \%$ de las infecciones de orina fueron causadas por el germen Escherichia coli. Otros gérmenes detectados en los urocultivos pero con menor incidencia fueron Proteus, Klebsiella y Enterococcus faecalis. El 55.6\% de los pacientes presentaban reflujo unilateral 69 unidades afectas en lado izquierdo y 61 en lado derecho). 40 pacientes padecían reflujo bilateral $(44.4 \%$ con un IC $95 \%$ de $34.09-55.27)$. Con respecto a las 130 unidades renales globales, el $6.9 \%$ eran de grado I, $11.5 \%$ de grado II, $54.6 \%$ de grado III y $26.9 \%$ de grado IV. Los reflujos con grado $\mathrm{V}$ ipsilateral y un reflujo grado III o IV en el contralateral no se consideran en nuestro estudio debido a que fueron intervenidos quirúrgicamente en su totalidad. La gran mayoría de los pacientes de nuestro estudio eran niñas con reflujo de grado III izquierdo. Sólo hemos considerado los reflujo de grado I y II si se asociaban a RVU contralateral de grados III o IV.

Cuando estratificamos los pacientes de nuestra serie en función del grado de dilatación ureteral medido de forma aislada independientemente del grado según la $\mathrm{Cl}$, observamos que $55.6 \%$ de los casos se clasificaron como leves, $32.2 \%$ como moderados y $12.2 \%$ como severos. Si bien existe una cierta relación entre el grado y la dilatación del uréter, más de la 1/3 parte de los casos catalogados como grado IV tenían una leve dilatación del uréter pero una gran dilatación de pelvis y cálices (Figura 1).

La nefropatía inicial por reflujo en el momento del diagnóstico valorada por DMSA se consideró leve en el $47.8 \%$ de los pacientes, moderada en el $14.4 \%$ y severa en el $8.9 \%$. 26 pacientes no tenían cicatrices renales en la presentación de la enfermedad. La gran mayoría de los pacientes con diagnóstico prenatal y nefropatía padecían una lesión difusa 


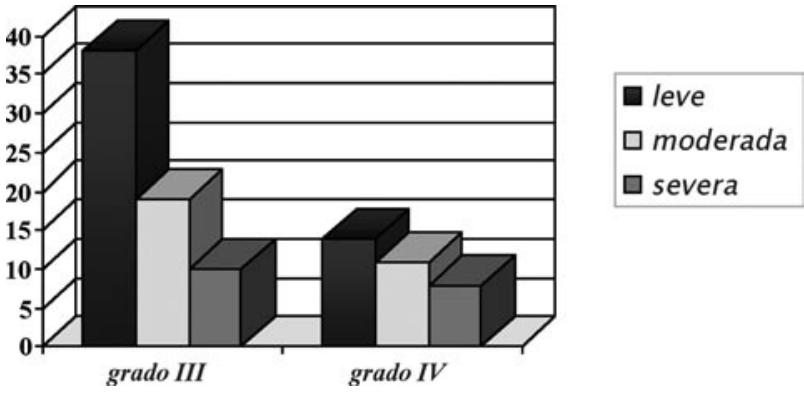

FIGURA 1. Dilatación ureteral aislada según el grado de reflujo.

del parénquima renal. A mayor edad, la nefropatía más característica es de tipo focal (polar superior o inferior). En pacientes con edades superiores a 10 años, la evolución de la enfermedad caracteriza la distribución difusa de la misma. Es muy significativo el hecho de que la gran mayoría de pacientes de más de 3 años tengan algún tipo de lesión cicatricial en la gammagrafía. Por el contrario, casi el $50 \%$ de los lactantes no tenían nefropatía en el momento del diagnóstico (Figura 2).

En nuestra serie el daño renal ha progresado tras el tratamiento en 18 pacientes $(20 \%)$. La nefropatía ha mejorado en el $14.4 \%$ y no se ha modificado en el 35,6\%. 27 pacientes permanecen sin lesión en el DMSA realizado 1 año después del tratamiento endoscópico. Esto supone que en el $80 \%$ de los pacientes la nefropatía por reflujo no ha progresado. 4 de los pacientes de la serie desarrollaron Insuficiencia Renal durante el seguimiento (media 4 años de evolución). Todos estos pacientes tenían más de 8 años y una historia de ITU recurrentes que habían sido tratadas en ciclos cortos de antibioterapia.

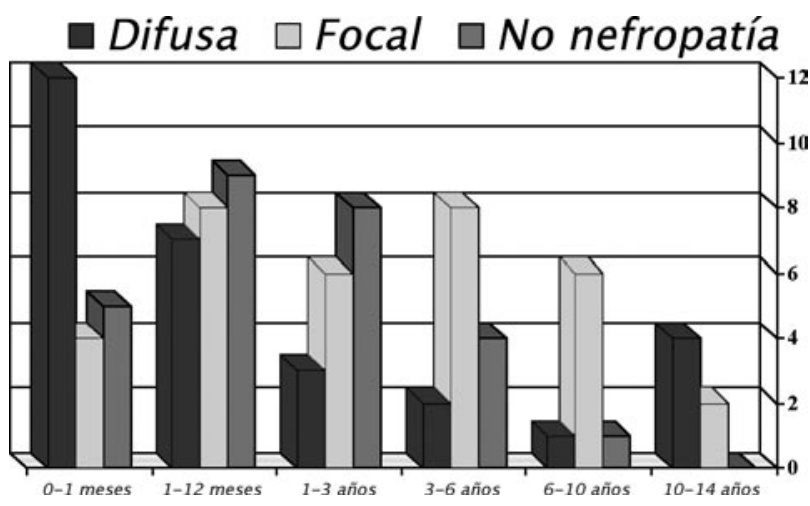

FIGURA 2. Nefropatía inicial según DMSA por segmentos de edad.
2 pacientes tuvieron recurrencia del reflujo (grado III) al cabo de 1 año de la inyección (ambos tenían disfunción miccional). No encontramos obstrucciones ureterovesicales post implante en nuestra serie. La nefrectomía fue el tratamiento final en 3 unidades renales de grado IV y dilatación ureteral severa.

Relacionando el sexo con el momento del diagnóstico (antenatal o postnatal) encontramos que en nuestro estudio existe una relación estadísticamente significativa $(p<0.001)$ entre sexo varón y diagnóstico antenatal. Esto quiere decir que los pacientes varones tienen 5.2 veces más posibilidades de ser diagnosticados antenatalmente que las niñas. Es más frecuente que el reflujo sea unilateral en los casos de diagnóstico fetal si bien esta relación no guarda significación estadística $(p=0.867)$. La mayoría de los casos en los que existe disfunción miccional durante la evolución, son de diagnóstico postnatal $(p=0.057)$. Aquellos pacientes que se diagnostican antes del nacimiento tienen menos posibilidades de padecer disfunción miccional junto con su reflujo vesicoureteral. Relacionando la existencia de disfunción miccional con la edad encontramos una relación entre la edad > 4 años y la incidencia de disfunción miccional. De la misma forma el número de infecciones de orina durante el seguimiento se relaciona directamente con el diagnóstico de disfunción miccional ( $p<0.001$ ) (Figura 3). La disfunción miccional es más probable en pacientes con edades mayores y en aquellos con mayor incidencia de ITU. En nuestra serie existe más riesgo de padecer nefropatía por

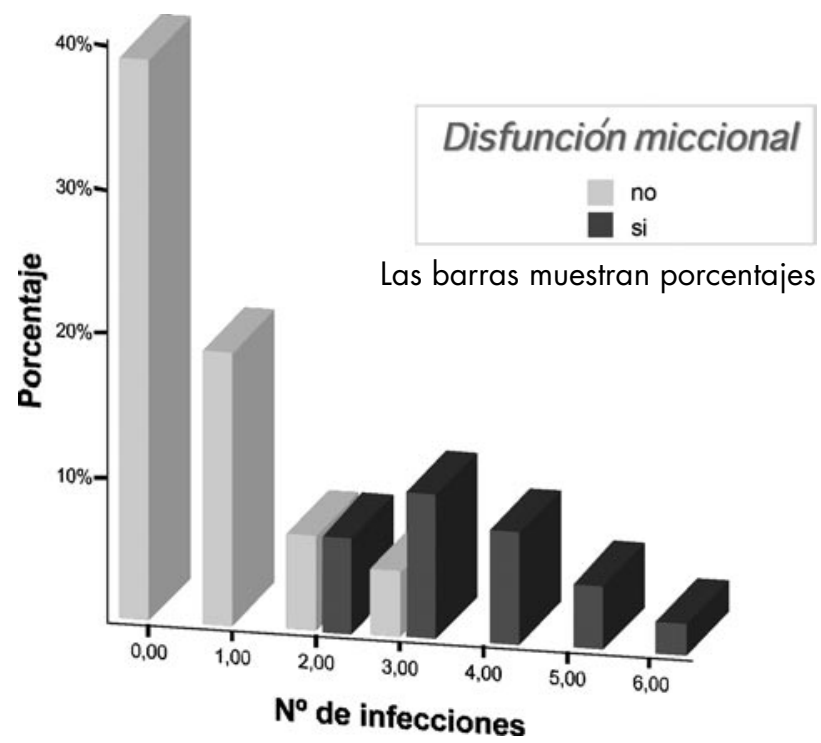

FIGURA 3. Relación entre disfunción miccional y número de infecciones. 
reflujo en aquellos pacientes que tienen disfunción miccional (es 4.5 veces más probable). Es decir, la ausencia de nefropatía es más probable si no existe disfunción miccional $(p=0.01)$. Ajustando por edad y número de infecciones en una regresión logística múltiple, comprobamos que la variable "diagnóstico prenatal" no influye de forma significativa para predecir disfunción miccional. Sin embargo el número de infecciones se convierte en la variable con mayor fuerza en relación con la existencia de disfunción miccional $(p<0.001)$ seguido por la edad al diagnóstico $(p=0.081)$. No hemos encontrado diferencias significativas entre el diagnóstico antenatal y la posibilidad de padecer nefropatía por reflujo en el momento del diagnóstico inicial $(p=0.784)$. Si bien la media de edad es superior en aquellos casos con nefropatía (1.7 años con respecto a 3.2 años), la mediana es igual en ambos grupos ( $p=0.323$ ) y ello condiciona la falta de significación estadística. Pese a ello los datos indican que la incidencia de nefropatía aumenta discretamente con la edad. Hemos encontrado diferencias claramente significativas $(p=0.015)$ en la relación entre bilateralidad y nefropatía por reflujo, lo que supone que existe 3.47 veces más riesgo de padecer nefropatía por reflujo en caso de que el reflujo afecte a ambos riñones que si la afectación es únicamente unilateral.

Ajustando por edad, bilateralidad y disfunción miccional en una regresión logística múltiple, comprobamos que la variable "edad al diagnóstico" no influye de forma significativa para predecir el riego de padecer nefropatía ( $p=0.232$ ). La variable más relevante es la bilateralidad (los pacientes con reflujo bilateral tienen 4 veces más probabilidades de padecer nefropatía por reflujo), con una significación de $p=0.067$ seguida por la existencia de disfunción miccional $(p=0.011)$.

No hemos encontrado diferencias estadísticamente significativas entre el sexo del paciente y el éxito de la primera inyección endoscópica $(p=0.432)$. Hemos encontrado diferencias $(p=0.046)$ en la relación entre bilateralidad y curación tras la $1^{\underline{a}}$ inyección les 2.3 veces más probable la resolución del reflujo en el primer intento si éste es unilateral y no afecta al sistema renal contralateral) (Tabla I).

Existe una estrecha relación entre la disfunción miccional y la posibilidad de curación del reflujo. Es 3.6 veces más probable la persistencia del reflujo tras la $1^{a}$ inyección si existe disfunción miccional concomitante. La significación según el $\chi^{2}$ de Pearson es $\mathrm{p}=0.007$.

La curación en el primer intento es 6.3 veces más probable en aquellos pacientes en los cuales no existe nefropatía por reflujo (cicatrices renales) en el momento del diagnóstico $(p=0.003)$. Existe una estrecha asociación estadística entre la dilatación ureteral aislada categorizada de forma independiente del grado y la posibilidad de resolución con la primera inyección $(p<0.001)$. Así, el $92 \%$ de aquellos sistemas calificados como "dilatación ureteral leve" se curaron tras la $1^{\underline{a}}$ inyección mientras que ninguno de aquellos juzgados como "dilatación ureteral severa" se resolvió al primer intento. Pero además, si relacionamos el grado de dilatación aislado del uréter respecto a las posibilidades de curación de la primera y la segunda inyección sumadas, vemos como esta variable mantiene una fuerte relación estadística: el $90.9 \%$ de aquellos sistemas calificados como severos no se curaron tras dos intentos mientras que el $100 \%$ de aquellos clasificados como leves sí lo hicieron $(p<0.001)$. Estratificando la variable en dicotómica con 2 categorías (leve y moderada-severa), apreciamos que es 20 veces más probable la curación si el uréter tiene una dilatación leve respecto a una dilatación severa o moderada $(p<0.001)$.

Ajustando la variable "curación tras la $1^{\underline{a}}$ inyección" por existencia de bilateralidad, disfunción miccional, nefropatía y dilatación ureteral aislada en una regresión logística múltiple, comprobamos que la variable más relevante es la dilatación ureteral medida de forma aislada independientemente del grado (los pacientes con dilatación moderada o severa tienen 22 veces menos probabilidades de ser curados con la primera inyección endoscópica), con una significación de $p<0.001$ seguida por la existencia de nefropatía y la bilateralidad.

Cuando analizamos de forma independiente el éxito de la terapia endoscópica tras la $2^{\underline{a}}$ inyección en aquellos casos en los que fracasó la terapia inicial, comprobamos que tanto la bilateralidad $(p=0.024)$ como la presencia de disfunción miccional $(p=0.021)$ son factores que se asocian a fracasos repetidos. La existencia de nefropatía en el momento del diagnóstico por el contrario no se asocia a probabilidad mayor de fracaso (Tabla II).

La bilateralidad del reflujo condiciona significativamente la progresión de la nefropatía tras el tratamiento endoscópico ( $p=0.034)$. Es 3.14 veces más probable que el daño renal empeore en aquellos paciente en los que son refluyentes ambas unidades renales. Aunque el hecho de que el diagnóstico del paciente haya sido antenatal parece que puede condicionar la mayor frecuencia de progresión de la nefropatía, este dato no es significativo $(p=0.618)$.

Si analizamos la variable "grado de dilatación ureteral aislada" con la progresión del daño 
renal encontramos resultados sorprendentes: Ningún paciente con dilatación ureteral calificada como leve tuvo progresión de la lesión renal. Todos estos casos permanecían sin lesión o bien esta se mantenía invariable. La mejoría se certificó en un bajo porcentaje de casos (7\%). Por el contrario, casi la mitad de los pacientes con dilataciones moderadas o severas tuvieron un empeoramiento en la lesión renal medida por DMSA tras el tratamiento endoscópico $(p<0.001)$.

Igualmente encontramos una alta significación estadística en la relación entre la existencia de nefropatía en el momento del diagnóstico inicial y la probabilidad de que la lesión progrese tras el tratamiento. El daño renal sólo progresó en aquellos que tenían lesión previa, si bien sólo lo hizo en la tercera parte de los pacientes $(p=0.003)$.
Hemos encontrado en nuestra serie una clara significación entre padecer disfunción miccional y la posibilidad de progresión del daño renal $(p=0.053)$. Es 2.7 veces más probable que aparezcan nuevas cicatrices renales en la evolución tras el tratamiento endoscópico si los pacientes son diagnosticados de disfunción miccional durante su seguimiento clínico.

En todos los pacientes en los que se resuelve el reflujo tras la primera inyección endoscópica, la situación inicial del riñón medida por DMSA permanece estable o incluso mejora. Por el contrario, más de la mitad de los casos que habían tenido progresión del daño renal eran pacientes en los cuales el reflujo no se había solucionado tras la $1^{\underline{a}}$ inyección $(p<0.001)$.

\section{TABLA I. RELACIÓN ENTRE CURACIÓN TRAS LA $7^{\cong}$ INYECCIÓN Y SEXO, BILATERALIDAD, DISFUNCIÓN MICCIONAL, NEFROPATÍA, DILATACIÓN URETERAL Y MATERIAL EMPLEADO.}

\begin{tabular}{|c|c|c|c|c|c|c|c|}
\hline \multirow{3}{*}{$\begin{array}{l}\text { Variable } \\
\text { Sexo }\end{array}$} & \multicolumn{2}{|c|}{ Curación $\mathrm{l}^{\mathrm{a}}$ inyección } & \multicolumn{2}{|c|}{ NO curación $1^{a}$ inyección } & \multirow{5}{*}{$\begin{array}{l}\mathbf{p} \\
0.432\end{array}$} & \multirow{5}{*}{\begin{tabular}{|l} 
OR \\
0.68
\end{tabular}} & \multirow{5}{*}{$\begin{array}{l}95 \% \text { IC (OR) } \\
0.25 \\
(1.79)\end{array}$} \\
\hline & $n$ & $\%$ & $\mathrm{n}$ & $\%$ & & & \\
\hline & & & & & & & \\
\hline \multirow{2}{*}{$\begin{array}{l}\text { Niñas } \\
\text { Niños }\end{array}$} & 44 & $65.7 \%$ & 23 & $34.3 \%$ & & & \\
\hline & 13 & $56.5 \%$ & 10 & $43.5 \%$ & & & \\
\hline \multicolumn{5}{|l|}{ Bilateralidad } & \multirow{3}{*}{0.046} & \multirow{3}{*}{0.43} & \multirow{3}{*}{$\begin{array}{l}0.18 \\
(1.03)\end{array}$} \\
\hline \multirow{2}{*}{$\begin{array}{l}\text { Unilat } \\
\text { Bilat }\end{array}$} & 36 & $72 \%$ & 14 & $28 \%$ & & & \\
\hline & 21 & $52.5 \%$ & 19 & $47.5 \%$ & & & \\
\hline \multicolumn{5}{|l|}{ Disfun miccional } & \multirow{3}{*}{0.007} & \multirow{3}{*}{0.28} & \multirow{3}{*}{$\begin{array}{l}0.11 \\
(0.72)\end{array}$} \\
\hline $\mathrm{SI}$ & 12 & $42.9 \%$ & 16 & $57.1 \%$ & & & \\
\hline NO & 45 & $72.6 \%$ & 17 & $27.4 \%$ & & & \\
\hline Nefropatía & & & & & \multirow{4}{*}{0.003} & \multirow{4}{*}{0.16} & \multirow{4}{*}{$\begin{array}{l}0.04 \\
(0.58)\end{array}$} \\
\hline $\mathrm{SI}$ & 35 & $53.8 \%$ & 30 & $46.2 \%$ & & & \\
\hline \multirow[t]{2}{*}{ NO } & 22 & $88 \%$ & 3 & $12 \%$ & & & \\
\hline & $n$ & $\%$ & $\mathrm{n}$ & $\%$ & & & \\
\hline Dilat. ureteral & & & & & \multirow{4}{*}{$<0.001$} & \multirow{4}{*}{ - } & \multirow{4}{*}{ - } \\
\hline leve & 46 & $92 \%$ & 4 & $8 \%$ & & & \\
\hline \multirow{2}{*}{$\begin{array}{l}\text { moderada } \\
\text { severa }\end{array}$} & 29 & $37.9 \%$ & 18 & $62.1 \%$ & & & \\
\hline & 11 & $0 \%$ & 11 & $100 \%$ & & & \\
\hline \multicolumn{5}{|l|}{ Material } & \multirow{3}{*}{0.038} & \multirow{3}{*}{0.36} & \multirow{3}{*}{$\begin{array}{l}0.14 \\
(0.96)\end{array}$} \\
\hline \multirow{2}{*}{$\begin{array}{l}\text { Teflón } \\
\text { Macroplástico }\end{array}$} & 11 & $45.8 \%$ & 13 & $54.2 \%$ & & & \\
\hline & 46 & $69.7 \%$ & 20 & $30.3 \%$ & & & \\
\hline
\end{tabular}


Uno de los datos de contingencia quizás más complejos en el análisis es la relación entre el tratamiento médico antes de la inyección endoscópica y la aparición de nuevas cicatrices renales. En nuestro estudio hemos comprobado como es 2.42 veces más frecuente la progresión de la nefropatía en aquellos pacientes que antes del tratamiento endoscópico fueron sometidos a tratamiento médico durante un período prolongado de tiempo. Si bien este hecho no es estadísticamente significativo $(p=0.680)$, sí que debe ser tenido en cuenta en el análisis.

Ajustando la variable "progresión de la nefropatía" por bilateralidad, disfunción miccional y edad al diagnóstico mediante una regresión logística múltiple, comprobamos que todas las variables relacionadas son significativas a la hora de predecir la probabilidad de progresión de la nefropatía. El hecho de tener disfunción miccional implica el riesgo más alto de que el daño renal progrese (es 6 veces más frecuente en estos pacientes), con una significación de $p=0.008$. Con respecto a la edad al diagnóstico, comprobamos como la edad juega un papel protector respecto a la probabilidad de desarrollar nuevas cicatrices renales: a más edad menos posibilidades de progresión de la nefropatía ( $p=0.043)$. Igualmente la bilateralidad del reflujo implica que la progresión de la nefropatía va a ser 3 veces más probable en estos pacientes respecto a aquellos en los que el reflujo sólo afecta a un sistema $(p=0.055)$.

\section{DISCUSIÓN}

El advenimiento de la corrección endoscópica del Reflujo Vesicoureteral como tercera alternativa iniciado por Puri y O'Donnell en 1984 modificó de forma radical las propuestas terapéuticas dicotómicas existentes hasta ese momento (cirugía o profilaxis). Pero no ha sido hasta la década de los 90 del pasado siglo cuando se generalizó su uso por la mayoría de las Unidades de Urología Pediátrica de todo el mundo (excepto en Estados Unidos, en donde sólo ha sido aceptado por la FDA el uso de Deflux® desde septiembre de 2001) (www.fda.gov/ $c d r h / p d f / p 000029 a . p d f)$ La inyección endoscópica subureteral de materiales biocompatibles se ha convertido en una alternativa establecida frente a la

\section{TABLA II. RELACIÓN ENTRE CURACIÓN TRAS LA 2ª INYECCIÓN Y SEXO, BILATERALIDAD, DISFUNCIÓN MICCIONAL, NEFROPATIA Y DILATACIÓN URETERAL AISLADA.}

\begin{tabular}{|c|c|c|c|c|c|c|c|}
\hline Variable & Curo & inyección & $\mathrm{NO} \mathrm{cl}$ & $2^{\mathrm{a}}$ inyección & p & OR & $95 \%$ IC (OR) \\
\hline \multirow[b]{2}{*}{ Sexo } & $n$ & $\%$ & $\mathrm{n}$ & $\%$ & \multirow{4}{*}{$\begin{array}{l}0.71 \\
\text { Fisher }\end{array}$} & \multirow{4}{*}{1.5} & \multirow{4}{*}{$0.30(7.36)$} \\
\hline & & & & & & & \\
\hline \multirow{2}{*}{$\begin{array}{l}\text { Niñas } \\
\text { Niños }\end{array}$} & 14 & $60.9 \%$ & 9 & $39.1 \%$ & & & \\
\hline & 7 & $70 \%$ & 3 & $30 \%$ & & & \\
\hline \multicolumn{5}{|l|}{ Bilateralidad } & \multirow{3}{*}{0.024} & \multirow{3}{*}{0.15} & \multirow{3}{*}{$0.03(0.86)$} \\
\hline $\mathrm{NO}$ & 12 & $85.7 \%$ & 2 & $14.3 \%$ & & & \\
\hline SI & 9 & $47.4 \%$ & 10 & $52.6 \%$ & & & \\
\hline Disfun miccional & & & & & \multirow{3}{*}{0.021} & \multirow{3}{*}{0.17} & \multirow{3}{*}{$0.03(0.82)$} \\
\hline $\mathrm{NO}$ & 14 & $82.4 \%$ & 3 & $17.6 \%$ & & & \\
\hline $\mathrm{SI}$ & 7 & $43.8 \%$ & 9 & $56.3 \%$ & & & \\
\hline \multicolumn{5}{|l|}{ Nefropatía } & \multirow{3}{*}{$\begin{array}{l}0.284 \\
\text { Fisher }\end{array}$} & \multirow{3}{*}{-} & \multirow{3}{*}{-} \\
\hline $\mathrm{NO}$ & 3 & $100 \%$ & 0 & $0 \%$ & & & \\
\hline $\mathrm{SI}$ & 18 & $60 \%$ & 12 & $40 \%$ & & & \\
\hline \multicolumn{5}{|l|}{ Dilat. ureteral } & \multirow{3}{*}{$\begin{array}{l}0.271 \\
\text { Fisher }\end{array}$} & \multirow{3}{*}{-} & \multirow{3}{*}{-} \\
\hline leve & 4 & $100 \%$ & 0 & $0 \%$ & & & \\
\hline moderada-severa & 17 & $58.6 \%$ & 12 & $41.4 \%$ & & & \\
\hline
\end{tabular}


profilaxis antibiótica prolongada y el tratamiento quirúrgico en el abordaje del RVU. Las tasas de curación obtenidas en las diferentes series publicadas varían en función del grado de reflujo y del material empleado. El Teflón es el material que mejores cifras ha conseguido hasta el momento $75.3 \%$ después de la primera inyección en un estudio multicéntrico sobre 9226 unidades ureterales) (9). El colágeno consigue curaciones del $65-70 \%$ pero con un alto índice de recurrencias por sus propiedades reabsortivas (10). El empleo de Macroplastico ${ }^{\circledR}$ (PDMS) logra resolver el reflujo en el $60-80 \%$ de los uréteres si bien no existen estudios a largo plazo que confirmen la veracidad de estos datos. El más reciente Deflux ${ }^{\circledR}$ obtiene una tasa del $68 \%$ en curaciones pero con un índice de recurrencias del $10 \%$ a 1 año (11).

Aunque en nuestro trabajo nos hemos centrado en los factores clínicos implicados en el éxito del tratamiento endoscópico, otros autores han intentado buscar parámetros propios de la técnica de inyección per se para justificar los fracasos terapéuticos. Los intentos de relacionar el sitio exacto de la inyección, el volumen adecuado del material y el desplazamiento del implante con la tasa de fracasos han resultado infructuosos y no significativos (12). En una revisión reciente acerca de las causas del fracaso en el empleo de Deflux ${ }^{\circledR}$, se citan entre otros: meato en "agujero de golf", duplicidad ureteral, atrofia renal severa, vejiga neurógena, poca experiencia del cirujano, irritación vesical, mala colocación del material y escaso volumen de inyección (13). Aunque no se hace referencia al estudio estadístico realizado, el autor no aporta explicaciones razonables que puedan justificar los resultados.

Las líneas de orientación proporcionadas por el grupo de expertos en RVU pediátrico de la American Urological Association (1997) recomendaron el desarrollo de técnicas de cistouretrografía que resultasen en la menor dosis posible de exposición radiológica (14). El desarrollo de los medios de contraste ecográfico de $2^{\underline{a}}$ generación estables como el Levograf $($ galactosa + ácido palmítico) persiguen este objetivo. El método ideal para el diagnóstico y seguimiento del RVU en niños debería cumplir una serie de requisitos como ser fiable, seguro, con la menor dosis posible de radiación, bien tolerado y no invasivo. La ecocistografía cumple casi todas estas premisas.

A la hora de valorar el cambio en el protocolo del diagnóstico del RVU debemos exigir a la nueva técnica una sensibilidad alta, además de un VPN lo más próximo posible al $100 \%$. Así nos aseguraremos que la posibilidad de que no diagnostiquemos mediante esta técnica un RVU es remota. En todos los estudios que analizaron los resultados de esta técnica hemos hallado VPN entre 91 y $100 \%$ mientras que la sensibilidad fluctúa entre $79-100 \%$ y la especificidad entre $86-97 \%$. Basándonos en estos criterios, en la actualidad en nuestro centro se emplea la sonocistografía como método standard de diagnóstico y seguimiento de los pacientes con RVU. La realización de CUMS convencional ha disminuido en nuestro centro en aproximadamente el $80 \%$ en los últimos 2 años.

Somos conscientes de que el cambio en la modalidad diagnóstica en un grupo de los pacientes de nuestra serie es susceptible de ser criticado, pero la considerable mejora respecto a técnicas convencionales (ausencia de radiación, sesión radiológica única) y la posibilidad de obtener los mismos resultados en nuestras clasificaciones hizo que consideráramos a todos los pacientes como una serie homogénea y sin diferencias en el método diagnóstico empleado.

El uso generalizado y sistematizado de la ecografía prenatal para el control del embarazo ha dado lugar a un considerable aumento del número de neonatos que son diagnosticados de anomalías del sistema urinario antes de que presenten sintomatología clínica. Se estima que aproximadamente un 10$25 \%$ de las dilataciones renales prenatales se deben a la existencia de Reflujo Vesicoureteral $(15,16)$. La ecografía de control postnatal a veces no proporciona la sospecha clínica necesaria para realizar una CUMS: hasta un $25 \%$ de los pacientes con hidronefrosis antenatal y ecografía postnatal normal tenían RVU en el estudio de Zerin, por lo que es recomendable que ante cualquier dilatación renal antenatal se realice una cistografía de screening al nacimiento (15). Pero lo realmente importante del RVU diagnosticado en ecografía gestacional es que su historia natural difiere respecto a la del RVU diagnosticado tras el nacimiento por una ITU (17). Esta enfermedad "diferente" se caracteriza por una gran predominancia masculina en su diagnóstico $180 \%$ son varones según la literatura). Este dato es coherente con los resultados de nuestro estudio, en el que si bien el número de niñas diagnosticadas de RVU tras hidronefrosis antenatal es similar al de niños, los varones presentan 5.2 veces más probabilidades de ser diagnosticados antenatalmente que las niñas (el $47.8 \%$ de los niños de nuestra serie se diagnosticaron antes del nacimiento frente a sólo el $14.9 \%$ de las niñas). La mayoría de los trabajos publicados con referencia al RVU de diagnóstico antenatal refieren una tasa de curación para los grados III a V del $36 \%(4.4 \%$ a $56 \%$ ) a los 2 años con profilaxis antibiótica como único tratamiento (19-23). Pero además, el $50 \%$ de estos pacientes con RVU de alto grado diagnosticado en ecografía prenatal muestran una mejoría en el grado a los 16 meses del nacimiento si son tratados 
médicamente (17). Lamentablemente en nuestra serie no podemos obtener estas cifras ya que independientemente del momento del diagnóstico, todos los pacientes siguieron la misma actitud terapéutica según su estadiaje y desconocemos como sería la evolución natural si no fuesen corregidos endoscópicamente.

Uno de los hallazgos más relevantes de nuestro trabajo ha sido la tipificación de la nefropatía encontrada en pacientes con diagnóstico prenatal. Aunque era más probable la existencia de nefropatía en casos antenatales, esta relación no guardaba significación estadística. Pero lo realmente importante era el tipo de daño renal: aquellos pacientes con diagnóstico antenatal tenían una lesión difusa frente a la nefropatía focal que caracterizaba a los que se diagnosticaron tras el nacimiento después de una ITU. La incidencia de lesión difusa fue decreciendo a medida que la edad al diagnóstico disminuía. Este hecho adquiere una importancia esencial si consideramos este tipo de reflujo como una enfermedad diferente con una fisiopatología diferente: existe una lesión renal antes del nacimiento no condicionada por las infecciones sino que es debida a la existencia de una displasia del riñón en formación por una anomalía en la nefrogénesis que altera también la unión ureterovesical (24).

Es posible que ante estas características diferenciales, los reflujos de diagnóstico antenatal deban ser abordados de una forma distinta: probablemente durante los 2 primeros años de vida el tratamiento más idóneo sea expectante esperando su resolución espontánea, con profilaxis antibiótica, tratamiento de la constipación, asegurando un buen vaciado vesical, evitando infecciones en relación con flora prepucial y con alimentación preferentemente por lactancia materna. A los 16-24 meses se deben reevaluar para plantear una opción diferente en función de la progresión del daño renal y el grado del reflujo (25).
Los pacientes con coexistencia de reflujo vesicoureteral y disfunción miccional se caracterizan por la alta incidencia de infecciones urinarias y las bajas tasas de curación espontánea (Tabla III).

Debido a estas circunstancias, es frecuente que estos pacientes sean candidatos con frecuencia a tratamiento quirúrgico de su reflujo. Pero la reimplantación, en muchos casos no soluciona por completo el problema y el reflujo tiene tendencia a perpetuarse y recidivar, padeciendo múltiples ITU durante la evolución (26). El reconocimiento de la constelación de síntomas que caracterizan el llamado "Dysfunctional Elimination Syndrom" (DES) es crucial para un adecuado tratamiento de estos pacientes (27). Esta secuencia de síntomas se caracteriza por frecuencia miccional, urgencia, inestabilidad vesical, constipación, enuresis, polaquiuria e infecciones recurrentes. Puede afectar incluso al $50-80 \%$ de los pacientes con RVU (28). Los pacientes con este síndrome DES se asocian a recurrencias en el reflujo, malos resultados post-reimplantación y tendencia a las ITU durante el seguimiento.

El análisis de nuestra serie con respecto a la disfunción miccional nos da cifras reveladoras de la importancia de este diagnóstico: el $31 \%$ de los pacientes de nuestra serie padecían algún tipo de disfunción miccional (DES). Estos pacientes padecían una media de 3.5 infecciones urinarias frente a las 0.6 del grupo sin DES; además, mostraban una probabilidad 4.5 veces superior al grupo sin disfunción de padecer nefropatía en el momento del diagnóstico y sus posibilidades de curación eran 3.6 veces menores tras la primera inyección endoscópica. La progresión del daño renal fue 2.7 veces más probable en el grupo de pacientes con DES ( $p=0.053)$ siendo la variable que más valor predictivo tenía a la hora de considerar la posibilidad de progresión de la nefropatía. La suma de estos datos aporta un

TABLA III. RESOLUCIÓN DEL RVU EN PACIENTES CON DISFUNCIÓN MICCIONAL.

\begin{tabular}{|cccc|}
\hline Autores & Pacientes & Grado reflujo & Resolución reflujo \\
\hline Koff \& Murtagh (1983) & 21 & III-IV & $43 \%$ \\
Nasrallah \& Simon (1984) & 26 & III-IV & $15 \%$ \\
Seruca (1989) & 31 & III-IV & $87 \%$ \\
Scholtmeijer et al (1994) & 22 & III-IV & $40 \%$ \\
IRSC (1992) & 155 & III-IV & $16 \%$ \\
\hline
\end{tabular}


panorama que, si bien es reconocido por otros autores, sorprende si analizamos únicamente pacientes con RVU tratados endoscópicamente: la coexistencia de disfunción miccional (DES) en pacientes con RVU condiciona menor tasa de curaciones, más daño renal, más posibilidades de progresión del daño renal y mayor número de infecciones tras el tratamiento.

Por este motivo es esencial el rápido reconocimiento y tratamiento de esta patología. El uso habitual de anticolinérgicos como la oxibutinina para evitar la inestabilidad vesical debe ir acompañado de medidas de choque dirigidas a evitar la constipación, un régimen de hidratación adecuado, pautas de higiene perineal correctas (especialmente en niñas), antibioterapia profiláctica y el exhaustivo control del vaciado vesical evitando el residuo postmiccional $(26,29)$. Es posible que los mejores resultados de la terapia endoscópica en pacientes con DES se logren tras un período mínimo previo de 6 meses de tratamiento antibioterápico y anticolinérgico además de medidas de rehabilitación miccional (30).

\section{Factores pronósticos que condicionan el éxito del tra- tamiento endoscópico del reflujo vesicoureteral en nuestra serie}

\section{Disfunción miccional}

La disfunción miccional se asocia a una alta incidencia de infecciones urinarias durante la evolución. Incluso el número de infecciones guarda una relación significativa con el hecho de padecer DES. Quizás uno de los resultados más significativos del estudio sea la estrecha relación entre la aparición de daño renal en el momento del diagnóstico y la coexistencia de disfunción miccional. Pero además, la progresión de las cicatrices renales es mucho más probable si el paciente ha sido diagnosticado, durante el seguimiento, de DES. Esta variable es la que guarda una más estrecha relación entre las estudiadas como válidas para prever la progresión del daño renal. Un dato concordante con la mayoría de las series revisadas es la alta tasa de fracasos del tratamiento endoscópico en pacientes pediátricos con DES. La curación del reflujo tras la primera inyección en pacientes con DES en nuestra serie es inferior al $45 \%$. Estos porcentajes se mantienen tras el segundo intento de resolución endoscópica. Podemos concluir que el diagnóstico de disfunción miccional junto con el reflujo de grados III y IV tiene implicaciones pronósticas que condicionan el éxito de la corrección endoscópica. Si este síndrome no es adecuadamente tratado, es muy probable que el reflujo se perpetúe pese al tratamiento endoscópico, las infecciones de orina sean recurrentes y la lesión renal, en caso de existir previamente, progrese.

\section{Nefropatía}

La existencia de nefropatía renal en el momento del diagnóstico del reflujo tiene implicaciones pronósticas respecto a las posibilidades de curación endoscópica. Aunque el tipo de nefropatía (difusa o focal) no determina la evolución del reflujo, el diagnóstico de ésta hace que el índice de fracaso del primer intento de curación endoscópica disminuya hasta el $54 \%$ respecto al $88 \%$ de curaciones en pacientes sin nefropatía. Este dato no se recoge en la mayoría de las publicaciones revisadas entre las que hacen referencia al tratamiento endoscópico. La lesión renal es más probable si el diagnóstico del reflujo fue en época prenatal (aunque no es significativo) pero esta suma de factores no incide en la tasa de curaciones. La progresión de la nefropatía está condicionada por la existencia de daño renal previo, la bilateralidad y la disfunción miccional, pero además, como cabría esperar en función de los resultados de las series más amplias revisadas, el fracaso en el tratamiento endoscópico aumenta las posibilidades de que las cicatrices renales progresen. En nuestro estudio hemos encontrado un dato sorprendente discordante con las series de Smellie en relación con el tratamiento médico: aquellos pacientes en los cuales se realizó terapia conservadora prolongada previa al tratamiento endoscópico la progresión de la lesión renal era 2 veces más frecuente aunque sin ser significativo. Este dato viene a apoyar la idea propugnada por Puri y Granata de que ante pacientes en riesgo de sufrir daño renal por las características de su reflujo de alto grado, es preferible no intentar la terapia médica y actuar directamente inyectando sustancias por vía endoscópica. Varios estudios comparativos entre tratamiento quirúrgico y tratamiento médico cuando hablan de nefropatía citan otra variable cuantitativa: el crecimiento renal medido por ecografía. Nosotros consideramos que el daño renal es mejor cuantificado basándonos en las características gammagráficas y por ello no hemos incluido en nuestros resultados las mediciones renales. Estas medidas deben ser ponderadas de forma adecuada en tablas de crecimiento y están sujetas a variaciones individuales de difícil estandarización.

\section{Bilateralidad}

La bilateralidad en el reflujo es un factor condicionante del pronóstico. La bilateralidad condiciona la tasa de curaciones espontáneas del reflujo, pero además es claramente significativa su relación con la aparición de nefropatía. De los pacientes con RVU bilateral, el $85 \%$ tenían daño renal en el momento del diagnóstico frente al $60 \%$ de los que padecían un reflujo unilateral. La bilateralidad es el factor que más condiciona, de todos los estudiados, la posibilidad de padecer nefropatía por reflujo. La bilateralidad va a condicionar, además, una tasa de fracasos 
tras la inyección endoscópica del $47 \%$ frente a un $28 \%$ en los unilaterales. Otro de los datos significativos en el estudio de la bilateralidad del reflujo es la relación con la progresión del daño, ya que el $30 \%$ de los pacientes que tenían un RVU a ambos riñones desarrollaron una nueva lesión renal durante su seguimiento (frente al $10 \%$ en los unilaterales), mejorando las imágenes de cicatrices renales sólo en 2 pacientes con reflujo bilateral grado III. Debido al bajo índice de curaciones obtenido con el tratamiento medico en los reflujos bilaterales de alto grado que además presentan lesión renal, probablemente el tratamiento más adecuado debería ser quirúrgico con reimplantación de ambos uréteres. Esta decisión es controvertida y no existe un consenso en la literatura al respecto, pero los datos aportados por nuestro estudio vendrían a confirmarnos la validez de este protocolo.

\section{Grado de dilatación ureteral}

La dilatación del uréter medida de forma independiente al grado del reflujo es un factor pronóstico clave en la expectativa de curación del reflujo tratado endoscópicamente. Aunque el hecho de intentar diferenciar ambas clasificaciones es cuestionable, la diferente valoración obtenida en nuestro trabajo sobre los mismos pacientes evaluados por el mismo especialista sin considerar la pelvis ni los cálices renales viene a ratificar nuestra hipótesis. Su validez como factor pronóstico lo demuestra su estrecha relación con el índice de fracasos tras la $1^{\underline{a}}$ inyección: el $100 \%$ de los sistemas calificados como severamente dilatados no se curaron. También fracasó en el $62 \%$ de los moderadamente dilatados y en el $8 \%$ de los que tenían una dilatación leve. Estos porcentajes se mantienen cuando consideramos también la $2^{a}$ inyección de Macroplástico. Unificando todos los factores con implicaciones pronósticas respecto a la tasa de curaciones vemos como el grado de dilatación es el que posee mayor valor por encima incluso de la bilateralidad del reflujo, la existencia de nefropatía o la disfunción miccional. Este dato no ha sido previamente reportado ni descrito y por tanto es uno de los aspectos más relevantes de nuestro trabajo y que debe ser objeto de nuevos estudios. El valor del grado de dilatación ureteral guarda, además, una estrecha relación con las posibilidades de desarrollar nuevas cicatrices renales hasta el punto de que el $45 \%$ de los pacientes que fueron catalogados como moderada o severamente dilatados presentaban nuevas lesiones renales durante el seguimiento frente al $0 \%$ de aquellos estratificados como leves. Este dato junto con la tasa de curaciones obtenida con los sistemas con dilataciones moderadas-severas debe hacernos replantear el protocolo de tratamiento en estos pacientes. En nuestra experiencia, la reimplantación quirúrgica es la opción más adecuada para los sistemas con dilataciones severas, ya que es muy probable que no consigamos la curación endoscópica y ello contribuya a una demora en la corrección del reflujo con el consiguiente riesgo de desarrollar nuevas cicatrices renales.

\section{CONCLUSIONES}

Este trabajo representa un intento de análisis para el estudio del reflujo vesicoureteral primario de alto grado que nos permita una sistematización en su manejo. El futuro de esta línea de investigación probablemente nos depare resultados esperanzadores. Es necesario obtener nuevos índices pronósticos que nos puedan ayudar a establecer protocolos de tratamiento internacionalmente consensuados. En este sentido serán necesarios estudios de validación de la nueva variable descrita en nuestro trabajo: el grado de dilatación ureteral aislada del grado según la clasificación internacional. Es posible que llegando a catalogar los sistemas mediante estas dos gradaciones obtengamos mayor información respecto a la evolución en el tiempo de las unidades renales.

Aunque es preciso ser críticos con todas las técnicas novedosas hasta que no pasen el tamiz del tiempo, no debemos olvidar que la terapia endoscópica lleva usándose en niños casi 20 años y que existen seguimientos publicados de pacientes controlados por el grupo de Prem Puri durante más de 17 años (31). En este sentido la eficacia de este tratamiento ya ha sido más que establecida y ahora sólo precisa mejorar la técnica y los materiales empleados.

Finalmente podremos concluir que:

1. El tratamiento endoscópico del reflujo Vesicoureteral grados III y IV es una alternativa válida para su empleo en pacientes pediátricos ya que consigue obtener curaciones globales cercanas al $90 \%$.

2. El éxito en la curación endoscópica del RVU está condicionado por la bilateralidad, la presencia de daño renal en el momento del diagnóstico, la existencia de disfunción miccional, el material empleado y el grado de dilatación del uréter.

3. El grado de dilatación del uréter es determinante a la hora de predecir la curación del reflujo y constituye el factor con mayor valor predictivo. Además, guarda una relación directamente proporcional con la progresión de la nefropatía tras el tratamiento.

4. La disfunción miccional asociada condiciona la aparición de ITU y tiene un papel relevante en la aparición de cicatrices renales y en la progresión de la nefropatía. La coexistencia de disfunción miccional con el reflujo implica una menor tasa de curaciones y una evolución desfavorable. 
5. La nefropatía inicial se asocia a menos curaciones y mayor probabilidad de progresión del daño renal pese al tratamiento.

6. La sonocistografía constituye una prueba válida para el screening, diagnóstico y seguimiento de los pacientes con RVU y permite catalogar a los pacientes según la IC además de evaluar el grado de dilatación ureteral.

7. La existencia de un reflujo bilateral de grado III o IV asociado a severa dilatación del uréter posiblemente deba ser tratado mediante un procedimiento quirúrgico abierto debido a la alta tasa de fracasos que se obtiene con el tratamiento endoscópico.

\section{BIBLIOGRAFÍA y LECTURAS RECOMENDADAS ( ${ }^{*}$ lectura de interés $y^{* *}$ lectura fundamental)}

1. KRAMER, S.A.: "Vesicoureteral reflux". Clinical Pediatric Urology, 3rd ed. Edited by Kelalis PP, King LR, Belman AB. Philadelphia, WB Saunders, 441-499, 1994.

**2. PURI, P.: "Endoscopic correction of vesicoureteral reflux". Curr. Opin. Urol. 10: 593, 2000.

**3. ELDER, J.S.; PETERS, C.A.; ARANT, B.S. Jr. y cols.: "Pediatric vesicoureteral reflux guidelines panel summary report on the management of primary vesicoureteral reflux in children". J. Urol., 157: 1846, 1997.

**4. SMELLIE, J.M.; BARRATT, T.M.; CHANTLER, C. y cols.: "Medical versus surgical treatment in children with severe bilateral vesicoureteric and bilateral nephropathy: a randomised trial". Lancet 357: 1329, 2001.

5. SMELLIE, J.M.; TAMMINEN-MÖBIUS, T.; OLBING, H. y cols.: "Five-year study of medical or surgical treatment in children with severe reflux: radiological renal findings". Pediatr. Nephrol. 6: 223, 1992.

6. OLBING, H.; HIRCHE, H.; KOSKIMIES, O.: "Renal growth in children with severe vesicoureteral reflux: 10 -year prospective study of medical and surgical treatment: the International Reflux Study in Children (European branch)". Radiology, 216: 731, 2000.

7. OLBING, H.; CLAESSON, I.; EBEL, K.D. y cols.: Renal scars and parenchymal thinning in children with vesicoureteral reflux: a 5-year report of the International Reflux Study in Children (European branch)". J. Urol., 148: 1553, 1992.

8. CAPOZZA, N.; LAIS, A.; MATARAZZO, E. y cols.: "Influence of voiding dysfunction on the outcome of endoscopic treatment for vesicoureteral reflux. J Urol 168: 1695-1698; 2002

*9. PURI, P.; GRANATA, C.: "Multicentre survey of endoscopic treatment of vesicoureteric reflux using polytetrafluoroethylene". J. Urol., 160: 1007, 1998.

10. CAPOZZA, N.; PATRICOLO, M.; LAIS, A. y cols.: "Endoscopic treatment of vesico-ureteral reflux: twelve years experience". Urol. Int., 67: 228, 2001.

11. LÄCKGREN, G.; WAHLIN, N.; SKÖLDENBERG, E. y cols.: "Long-term followup of children treated with dextranomer / hyaluronic acid copolymer for vesicoureteral reflux". J. Urol., 166: 1887, 2001.

12. BOSTON, V.: "What factors cause failure and can these factors be predicted?". Read at the International Workshop on VUR, Dublin, Ireland, 6-7 September 2002.

13. LÄCKGREN, G.: "Long-term clinical results after treatment with Deflux in children with VUR and either double ureters or small kidneys". Read at the International Workshop on VUR, Dublin, Ireland, 6-7 September 2002.

*14. ELDER, J.S.; PETERS, C.A.; ARANT, B.S. Jr. y cols.: "Pediatric vesicoureteral reflux guidelines panel summary report on the management of primary vesicoureteral reflux in children”. J. Urol., 157: 1846, 1997.

15. ZERIN, J.M.: "Impact of contrast medium temperature on bladder capacity and cystographic diagnosis of vesicoureteral reflux in children". Radiology, 187: 161, 1993.

16. FINE, R.N.: "Diagnosis and treatment of fetal urinary tract abnormalities". J. Pediatr. 121: 333, 1992.

*17. FARHAT, W.; McLORIE, G.; GEARY, D. y cols.: "The natural history of neonatal vesicoureteral reflux associated with antenatal hydronephrosis". J. Urol., 164: 1057, 2000.

18. AVNI, E.F.; SCHULMAN, C.C.: "The origin of vesicoureteric reflux in male newborns: further evidence in favour of a transient urethral obstruction". Br. J. Urol ., 78: 454, 1996.

19. BURGE, D.; GRIFFITHS, M.; MALONE, P. y cols.: "Fetal vesicoureteral reflux: outcome following consecutive postnatal management". J. Urol., 148: 1743, 1992.

20. ANDERSON, P.A.; RICKWOOD, A.M.: "Features of primary vesicouretric reflux detected by prenatal sonography". Br. J. Urol., 67: 267, 1991.

*21. YEUNG, C.K.; GODLEY, M.L.; DHILLON, H.K. y cols.: "The characteristics of primary vesico-ureteric reflux in male and female infants with pre-natal hydronephrosis". Br. J. Urol., 80: 319, 1997.

22. STEELE, B.T.; ROBITAILLE, P.; DE MARIA, J. y cols.: "Follow-up evaluation of prenatally recognized vesicoureteric reflux". J. Pediatr., 115: 95, 1989.

23. HERNDON, C.D.; McKENNA, P.H.; KOLON, T.F. y cols.: "A multicenter outcomes analysis of patients with neonatal reflux presenting with prenatal hydronephrosis". J. Urol., 162: 1203, 1999.

24. RANSLEY, P.G.: "VUR Understanding \& Approach. Read at ESPU Course "Antenatally-diagnosed Hydronephrosis: pre \& postnatal assessment \& clinical approach”. Bodrum, Turkey, 10-11 May 2002.

25. MOURIQUAND, P.D.; WHITTEN, M.; PRACROS, J.P.: "Pathophysiology, diagnosis and management of prenatal upper tract dilation”. Prenat. Diagn., 21: 942, 2001.

26. HERNDON, A.; DE CAMBRE, M.; McKENNA, P.H.: "Changing concepts concerning the management of vesicoureteral reflux". J. Urol., 166: 1439, 2001.

27. KOFF, S.; WAGNER, T.; JAYANTHI, V.: "The relationship among dysfunctional elimination syndromes, primary vesicoureteric reflux and urinary tract infection in children". J. Urol., 60: 1019, 1998.

28. CHANDRA, M.; MADDIX, H.: "Urodynamic dysfunction in infants with vesicoureteral reflux". J. Pediatr., 136: 754, 2000.

29. SNODGRASS, W.: "The impact of treated dysfunctional voiding on the non-surgical management of vesicoureteral reflux". J. Urol., 160: 1823, 1998.

30. CAPOZZA, N.; LAIS, A.; MATARAZZO, E. y cols.: "Influence of voiding dysfunction in the outcome of endoscopic treatment of vesicoureteral reflux". Read at The National Conference of the American Academy of Pediatrics, Section of Urology, San Francisco, CA, USA, 20-22 October 2001.

*31. CHERTIN, B.; COLHOUN, E.; VELAYUDHAM, M. y cols.: "Endoscopic treatment of vesicoureteral reflux: 11 to 17 years of followup". J. Urol., 167: 1443, 2002. 